\title{
Slave-rotor mean field theories of strongly correlated systems and the Mott transition in finite dimensions
}

\author{
Serge Florens ${ }^{1,2}$ and Antoine Georges ${ }^{3,2}$ \\ ${ }^{1}$ Institut für Theorie der Kondensierten Materie, \\ Universität Karlsruhe, 76128 Karlsruhe, Germany \\ ${ }^{2}$ Laboratoire de Physique Théorique, Ecole Normale Supérieure, \\ 24 rue Lhomond, 75231 Paris Cedex 05, France \\ ${ }^{3}$ Centre de Physique Théorique, Ecole Polytechnique, 91128 Palaiseau Cedex
}

\begin{abstract}
The multiorbital Hubbard model is expressed in terms of quantum phase variables ("slave rotors") conjugate to the local charge, and of auxiliary fermions, providing an economical representation of the Hilbert space of strongly correlated systems. When the phase variables are treated in a local mean-field manner, similar results to the dynamical mean-field theory are obtained, namely a Brinkman-Rice transition at commensurate fillings together with a "preformed" Mott gap in the single-particle density of states. The slave- rotor formalism allows to go beyond the local description and take into account spatial correlations, following an analogy to the superfluid-insulator transition of bosonic systems. We find that the divergence of the effective mass at the metal- insulator transition is suppressed by short range magnetic correlations in finite- dimensional systems. Furthermore, the strict separation of energy scales between the Fermi- liquid coherence scale and the Mott gap, found in the local picture, holds only approximately in finite dimensions, due to the existence of low-energy collective modes related to zero-sound.
\end{abstract}

\section{INTRODUCTION}

Strongly correlated fermion systems constitute a challenge, both from a fundamental point of view (with phenomena such as the Mott transition [1] and high- temperature superconductivity), and on a more quantitative level with the need of reliable tools to handle intermediate and strong coupling regimes (even for simplified models such as the Hubbard model). In recent years, the dynamical mean-field theory (DMFT) has allowed for significant progress in this respect [2]. In particular, this approach has led to a detailed theory of the Mott transition, and to a quantitative description of the physics of strongly correlated metals. Despite these successes, the limitations of this approach have been emphasized on many occasions. The main one has to do with the effect of spatial correlations (e.g magnetic short-range correlations), and more precisely with the effect of these correlations on the properties of quasiparticles. For example, the tendency to form singlet bonds due to superexchange is widely believed to be a key physical effect in weakly doped Mott insulators. Also, at the technical level, the application of DMFT to materials with a large orbital degeneracy (e.g in combination with ab-initio methods $3,[4,5]$ ), as well as cluster extensions of DMFT [2, 6] are computationally challenging because they involve the solution of a multi-orbital quantum impurity model.

For these reasons, there is still a strong need for approximate, simpler treatments of strongly correlated fermion models. Those treatments should incorporate some of the DMFT successes (e.g regarding the description of the metal-insulator transition), but they should also pave the road for describing physical effects beyond DMFT at least at a qualitative level.
The purpose of this paper is to present a simple mean field description of correlated systems which fullfills some of these goals. Our main idea is to focus on the degrees of freedom associated to the relevant physical variable associated to the Mott transition, namely a slave quantum rotor field, dual to the local electronic charge. This slave rotor representation was introduced previously by us for the description of quantum impurity models and mesoscopic devices [7, [8], and is applied here in the context of lattice models. This allows for a simple reformulation of the orbitally degenerate Hubbard model, which, when the interaction has full orbital symmetry, is quite superior to previously developed slave- boson representations 9, 10, 11.

When the simplest possible (single-site) mean-field approximation is used in conjunction with this slave-rotor representation, a description of the Mott transition very similar to that of DMFT is found. The metallic phase disappears through a Brinkman-Rice transition, at which the quasiparticle wieight vanishes and the effective mass diverges. The slave rotor approach does preserve Hubbard bands in the insulator, and a "preformed" Mott spectral gap opening up discontinuously at the transition is found, as in DMFT.

The most interesting aspect of our approach lies however in the possibility of going beyond this purely local mean-field description. By decoupling the spinons and slave rotor degrees of freedom, the Hubbard model is mapped onto a free spinon hamiltonian selfconsistently coupled to a quantum XY lattice model. The (dis)ordering transition of the latter corresponds to the Mott transition, in analogy with the superfluid-insulator transition of the bosonic Hubbard model. Because spatial correlations are now included, we find important modifi- 
cations to the DMFT picture. In particular, the effective mass remains finite at the transition, due to the quenching of the macroscopic entropy by magnetic correlations in the Mott phase. Importantly, low-energy charge collective modes are shown to affect the opening of the Mott gap, which now develops in a continuous manner, so that the separation of energy scales found in DMFT only holds in an approximate manner. These simple results can be considered as deviations from the DMFT predictions that could possibly be observed in photoemission experiments [12]. However, restauration of the local gauge symmetry should occur due to fluctuations beyond the mean-field approximation, possibly modifying the latter result on a qualitative way.

The paper is organised as follows. In section III we introduce the exact slave rotor description of a simple atomic level with orbital degeneracy, and show that an approximate treatment of the local constraint is sufficient to describe correctly the full Coulomb staircase, as well as one-particle spectra. Then, in section III] we develop the simplest (local) mean field treatment of both the Anderson and Hubbard models, and in the latter case, study the multiorbital Mott transition. Finally, spatial fluctuations beyond DMFT are included in section IV] with an emphasis on the behavior of the effective mass and the excitation spectrum. The conclusion presents several possible applications and extensions of our formalism, and also discusses some of the open issues raised by our results.

\section{ROTOR REPRESENTATION OF INTERACTING FERMIONS}

\section{A. Slave-rotor representation}

In Ref. 7] (see also [13]), we introduced a representation of the Hilbert space of $N$ fermions $d_{\sigma}^{\dagger}$ in terms of a collective phase degree of freedom $\theta$, conjugate to the total charge, and of $N$ auxiliary fermions $f_{\sigma}^{\dagger}$. The spin/orbital index runs over $N$ values $\sigma=1 \ldots N$ (e.g $\sigma=\uparrow, \downarrow$ for $N=2$ ). In the following, we consider only interactions which have the full $S U(N)$ spin/orbital symmetry. Let us consider the Hamiltonian corresponding to a single "atomic level", in the presence of a local Hubbard repulsion:

$$
H_{\mathrm{at}}=\sum_{\sigma} \epsilon_{0} d_{\sigma}^{\dagger} d_{\sigma}+\frac{U}{2}\left[\sum_{\sigma} d_{\sigma}^{\dagger} d_{\sigma}-\frac{N}{2}\right]^{2}
$$

The crucial point is that the spectrum of the atomic Hamiltonian (11) depends only on the total fermionic charge $Q=0, \cdots, N$ and has a simple quadratic dependence on $Q$ :

$$
E_{Q}=\epsilon_{0} Q+\frac{U}{2}\left[Q-\frac{N}{2}\right]^{2}
$$

There are $2^{N}$ states, but only $N+1$ different energy levels, with degeneracies $\left(\begin{array}{l}N \\ Q\end{array}\right)$. In conventional slave boson methods 9, 11], a bosonic field is introduced for each atomic state $\left|\sigma_{1} \cdots \sigma_{Q}\right\rangle$ (along with spin-carrying auxiliary fermions $\left.f_{\sigma}^{\dagger}\right)$. Hence, these methods are not describing the atomic spectrum in a very economical manner, and lead to very tedious calculations when orbital degeneracy becomes large, even at the mean-field level [10, 11, 14, 15]. However, we stress that the extensive number of degrees of freedom necessary in those other approaches can become useful when the $S U(N)$ symmetry is broken, either by magnetic order or crystal fields.

The spectrum of (11) can actually be reproduced by introducing, besides the set of auxiliary fermions $f_{\sigma}^{\dagger}$, a single additional variable, namely the angular momentum $\hat{L}=-i \partial / \partial \theta$ associated with a quantum $O(2)$ rotor $\theta$, an angular variable in $[0,2 \pi]$. Indeed, the energy levels (2) can be obtained using the following Hamiltonian

$$
H_{\mathrm{at}}=\sum_{\sigma} \epsilon_{0} f_{\sigma}^{\dagger} f_{\sigma}+\frac{U}{2} \hat{L}^{2}
$$

A constraint must be imposed, which insures that the total number of fermions is equal to the $O(2)$ angular momentum (up to a shift, in our conventions):

$$
\hat{L}=\sum_{\sigma}\left[f_{\sigma}^{\dagger} f_{\sigma}-\frac{1}{2}\right]
$$

This restricts the allowed values of the angular momentum to be $\ell=Q-N / 2=-N / 2,-N / 2+1, \cdots, N / 2-$ $1, N / 2$, while in the absence of any constraint $\ell$ can be an arbitrary (positive or negative) integer. The spectrum of (3) is $\epsilon_{0} Q+U \ell^{2} / 2$, with $\ell=Q-N / 2$ thanks to (44), so that it coincides with (2).

It is easily checked that the full Hilbert space is correctly described as:

$$
\left|\sigma_{1} \ldots \sigma_{Q}\right\rangle_{d}=\left|\sigma_{1} \ldots \sigma_{Q}\right\rangle_{f}|\ell=Q-N / 2\rangle_{\theta}
$$

in which $\left|\sigma_{1} \ldots \sigma_{Q}\right\rangle_{d, f}$ denotes the antisymmetric fermion state built out of $d$ - and $f$-fermions, respectively, and $|\ell\rangle_{\theta}$ denotes the quantum rotor eigenstate with angular momentum $\ell$, i.e. $\langle\theta \mid \ell\rangle_{\theta}=e^{i \ell \theta}$. For $N=2$, this corresponds to: $|\uparrow\rangle_{d}=|\uparrow\rangle_{f}|0\rangle_{\theta},|\downarrow\rangle_{d}=|\downarrow\rangle_{f}|0\rangle_{\theta}$, $|\uparrow \downarrow\rangle_{d}=|\uparrow \downarrow\rangle_{f}|+1\rangle_{\theta}$ and $|0\rangle_{d}=|0\rangle_{f}|-1\rangle_{\theta}$. The creation of a physical electron with spin $\sigma$ is associated to the action of $f_{\sigma}^{\dagger}$ on such a state as well as raising the total charge (angular momentum) by one unit. Since the raising operator is $e^{i \theta}$, this leads to the representation:

$$
d_{\sigma}^{\dagger} \equiv f_{\sigma}^{\dagger} e^{i \theta}, d_{\sigma} \equiv f_{\sigma} e^{-i \theta}
$$

The key advantage of the quantum rotor representation is that the original quartic interaction between fermions has been replaced in (3) by a simple kinetic term for the phase field, $(U / 2) \hat{L}^{2}$.

We point out here that a similar phase representation was developed before in the context of Coulomb blockade in mesoscopic systems, see e.g [16, 17, 18]. However, 
the present work and our previous paper [7] present the first applications of the rotor technique to the context of strongly correlated lattice models. In particular, the question of quasiparticle coherence which is crucial to the description of a Fermi liquid cannot be investigated seriously with a phase-only description [19], as shown in [8]. In this perspective, the slave rotor should be seen as a natural extension (and simplification) of the usual slave boson techniques [20, 21] in the context of a finite but orbitally symmetric Coulomb repulsion. In principle, it can also be applied to systems with long-range interactions [13, 22].

\section{B. Treating the constraint on average: atomic limit}

In the following, we will study different kinds of meanfield approximations based on this slave-rotor representation. A common trait of these mean-field approximations is that the number constraint (4) will be treated on average. This is equivalent to treating the constraint in a "grand-canonical" ensemble, which would of course be exact in the limit of a large spin/orbital degeneracy $N \rightarrow \infty$. In this section, we investigate the accuracy of this approximation for the atomic Hamiltonian (1), for finite values of $N$.

\section{Coulomb staircase: occupancy vs. $\epsilon_{0}$}

Let us first consider the dependence of the average occupancy $\langle\hat{Q}\rangle=\left\langle\sum_{\sigma} d_{\sigma}^{\dagger} d_{\sigma}\right\rangle$ on the position of the atomic level $\epsilon_{0}$, which reads:

$$
\langle\hat{Q}\rangle_{a t}=\frac{1}{Z_{a t}} \sum_{Q=0}^{N}\left(\begin{array}{l}
N \\
Q
\end{array}\right) Q e^{-\beta E_{Q}}
$$

with $Z_{a t}=\sum_{Q}\left(\begin{array}{l}N \\ Q\end{array}\right) e^{-\beta E_{Q}}$. In the limit of zero temperature, the dependence of $\langle\hat{Q}\rangle$ on $\epsilon_{0}$ is the "Coulomb staircase" in Fig. 1] When treating the constraint on average,

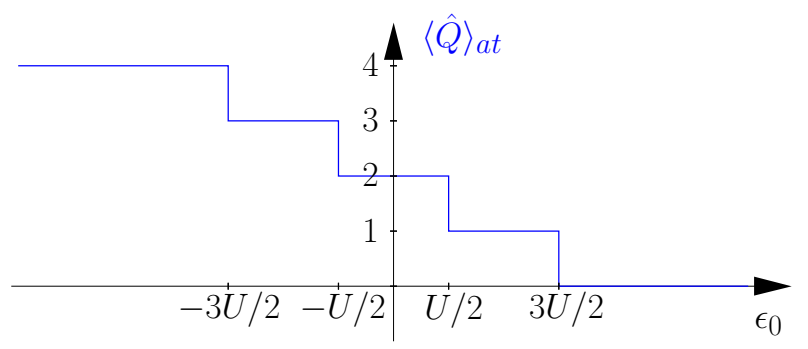

FIG. 1: Coulomb staircase in the atomic limit for the case of two orbitals, $N=4$.

a Lagrange multiplier $h$ is introduced which is conjugate to (4), and one optimizes over $h$ instead of fully integrating over it. This amounts to consider the following effective Hamiltonians:

$$
\begin{aligned}
& H_{f}^{a t}=\left(\epsilon_{0}-h\right) \sum_{\sigma} f_{\sigma}^{\dagger} f_{\sigma} \\
& H_{\theta}^{a t}=\frac{U}{2} \hat{L}^{2}+h\left(\hat{L}-\frac{N}{2}\right)
\end{aligned}
$$

The Lagrange multiplier $h$ is determined by the average constraint equation:

$$
\langle\hat{L}\rangle_{h}=N\left[n_{F}\left(\epsilon_{0}-h\right)-\frac{1}{2}\right]
$$

in which $\langle\hat{L}\rangle_{h}$ is the average of $\hat{L}$ in the Hamiltonian (9):

$$
\langle\hat{L}\rangle_{h}=\frac{1}{Z_{\theta}} \sum_{\ell=-\infty}^{+\infty} \ell e^{-\beta E_{\ell}}
$$

with: $E_{\ell}=U \ell^{2} / 2+h \ell$ and $Z_{\theta}=\sum_{\ell} e^{-\beta E_{\ell}}$. Solving (10) for $h$ as a function of $\epsilon_{0}$ and temperature $T=1 / \beta$ yields the dependence of the total charge within this approximation:

$$
\langle\hat{Q}\rangle=N n_{F}\left[\epsilon_{0}-h\left(\epsilon_{0}, T\right)\right]
$$

We need to compare this approximation to the exact result (7) in the atomic limit. A graphical representation (Fig. 2) is useful in order to understand the solution of (10). At $T=0$, one finds $h=\epsilon_{0}$, as long as $0<Q<N$.

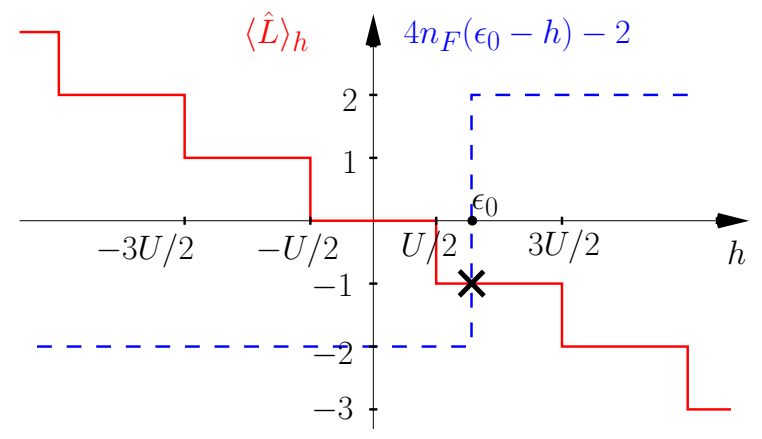

FIG. 2: Graphical solution of the average constraint equation (10). The intersect (cross) moves exactly along the Coulomb staircase shown in figure 1

The exact dependence of the average charge $Q$ upon $\epsilon_{0}$ at $T=0$ is correctly reproduced by our approximation, corresponding to the "Coulomb staircase":

$$
Q=\frac{N}{2}+\ell \text { for }-U \frac{2 \ell+1}{2}<\epsilon_{0}<-U \frac{2 \ell-1}{2}
$$

Note that $h-\epsilon_{0}$ vanishes linearly with temperature according to: $h=\epsilon_{0}-T \ln (N / Q-1)+\cdots$ : this is why the full Coulomb staircase can be reproduced with a single Fermi factor in (12). At finite temperature, our approximation does not coincide with the exact result for $Q_{a t}\left(\epsilon_{0}, T\right)$, but deviations are only sizeable for temperatures comparable to $U$, which is not a severe limitation in practice. 


\section{Spectral functions}

We now study the consequences of the approximate treatment of the constraint for the Green's function and spectral function. Following (89), the quantum rotor and auxiliary fermion degrees of freedom are described by two independent Hamiltonians, so that the Green's function of the physical electron $G_{d}(\tau) \equiv-\left\langle T d_{\sigma}(\tau) d_{\sigma}^{\dagger}(0)\right\rangle$ factorizes into:

$$
G_{d}(\tau)=G_{f}(\tau) G_{\theta}(\tau)
$$

with $G_{\theta}(\tau) \equiv\langle\exp i[\theta(0)-\theta(\tau)]\rangle$. Equivalently, the physical electron spectral function is given by:

$\rho_{d}(\omega)=-\int d \omega^{\prime} \rho_{f}\left(\omega^{\prime}\right) \rho_{\theta}\left(\omega-\omega^{\prime}\right)\left[n_{F}\left(-\omega^{\prime}\right)+n_{B}\left(\omega-\omega^{\prime}\right)\right]$

Let us consider $T=0$, and $\epsilon_{0}$ in the range corresponding to the plateau of charge $Q$ in the Coulomb staircase. The ground-state energy is $E_{Q}=U(Q-N / 2)^{2}+\epsilon_{0} Q$ and its degeneracy is $\left(\begin{array}{l}N \\ Q\end{array}\right)$. The two excited states obtained by adding or removing a particle correspond to transition energies: $\Delta_{ \pm}=E_{Q \pm 1}-E_{Q}= \pm \epsilon_{0} \pm U(Q-N / 2 \pm 1 / 2)$. When acting with $d_{\sigma}^{\dagger}$ on the ground-state, only those ground-state components which do not already contain $\sigma$ contribute, and there are $\left(\begin{array}{c}N-1 \\ Q\end{array}\right)=\left(1-\frac{Q}{N}\right)\left(\begin{array}{l}N \\ Q\end{array}\right)$ such components. Similarly, when acting with $d_{\sigma}$, only the components in which $\sigma$ is occupied contribute, and there are $\left(\begin{array}{l}N-1 \\ Q-1\end{array}\right)=\frac{Q}{N}\left(\begin{array}{l}N \\ Q\end{array}\right)$ of them. From these considerations, we see that the exact spectral function reads, at $T=0$ :

$$
\rho_{d}^{a t}(\omega)=\left(1-\frac{Q}{N}\right) \delta\left(\omega-\Delta_{+}\right)+\frac{Q}{N} \delta\left(\omega+\Delta_{-}\right)
$$

These two atomic transitions are the precursors of the Hubbard bands in the solid. Note that they have unequal weights, except at half-filling $Q=N / 2$. At finite temperature, additional peaks appear (except for $N=2$ ), corresponding to transition between two excited states (with exponentially small weight for $T \ll U$ ).

Remarkably, the expressions (1415) in which the quantum rotor and auxiliary fermions are treated as decoupled, do reproduce this exact result at $T=0$. The easiest way to see this is to notice that, at $T=0$, $G_{f}(\tau)=-(1-Q / N) \theta(\tau)+(Q / N) \theta(-\tau)$, since $n_{F}\left(\epsilon_{0}-\right.$ $h)=Q / N$. The rotor Green's function $G_{\theta}$ is $e^{-\Delta_{+} \tau}$ for $\tau>0$ and $e^{\Delta_{-} \tau}$ for $\tau<0$. Substituting into (14), this corresponds to the exact expression (16). Alternatively, one can use the expressions of the $T=0$ spectral functions into (15): $\rho_{f}(\omega)=\delta\left(\omega-\epsilon_{0}+h\right)$ and $\rho_{\theta}(\omega)=-\delta\left(\omega-\Delta_{+}\right)+\delta\left(\omega-\Delta_{-}\right)$, keeping in mind that $n_{F}\left(\epsilon_{0}-h\right)=Q / N$ while $n_{B}\left(\omega-\epsilon_{0}+h\right)=-\theta(-\omega)$ as $T \rightarrow 0$. Again, deviations between the approximate treatment and the exact results are found at finite temperature, but remain small for $T \ll U$. Let us emphasize that, because the rotor Green's function $G_{\theta}$ is a continuous function at $\tau=0$, with $G_{\theta}(\tau=0)=1$, the factorized approximation (14) insures that the physical (d-electron) spectral function is correctly normalized with total spectral weight equal to unity.

To summarize, we have found that treating the constraint on average reproduces accurately the atomic limit at $T=0$, both regarding the Coulomb staircase dependence of $Q$ vs. $\epsilon_{0}$, and regarding the spectral function. This is a key point for the methods introduced in this article, which allows them to describe reasonably the high energy features of strongly correlated systems.

\section{Functional integral formulation}

We briefly introduce here a functional integral formalism for the $f_{\sigma}^{\dagger}$ and $\theta$ degrees of freedom, and derive the action associated with (1). This is simply done by switching from phase and angular-momentum operators $(\hat{\theta}, \hat{L})$ to fields $\left(\theta, \partial_{\tau} \theta\right)$ depending on imaginary time $\tau \in[0, \beta]$, with $\theta(\beta)=\theta(0)+2 \pi n$. The action is constructed from $S \equiv \int_{0}^{\beta} d \tau\left[-i \hat{L} \partial_{\tau} \theta+H+f^{\dagger} \partial_{\tau} f\right]$, and an integration over $\hat{L}$ is performed. It is also necessary to introduce a Lagrange multiplier $\widetilde{h}$ in order to implement the constraint $\stackrel{L}{L}=\sum_{\sigma} f_{\sigma}^{\dagger} f_{\sigma}-N / 2$. We note that, because of the charge conservation on the local impurity, $\widetilde{h}$ can be chosen to be independent of time, with $\widetilde{h} \in[0,2 \pi / \beta]$. This leads to the following expression of the action:

$$
S_{\mathrm{at}}=\int_{0}^{\beta} \mathrm{d} \tau \sum_{\sigma} f_{\sigma}^{\dagger}\left(\partial_{\tau}+\epsilon_{0}+i \widetilde{h}\right) f_{\sigma}+\frac{\left(\partial_{\tau} \theta+\widetilde{h}\right)^{2}}{2 U}-i \frac{N}{2} \widetilde{h}
$$

The constraint is implemented exactly provided $\widetilde{h}$ is integrated over. The above approximation amounts to evaluate the integral by a saddle-point approximation over $\widetilde{h}$, and the saddle-point is found to be on the real axis, with $\widetilde{h}=i h$.

Finally, let us mention that, in a previous publication [7], we have explained in detail the connection between the rotor construction and the HubbardStratonovich decoupling of the interaction in the charge channel.

\section{THE SIMPLEST MEAN-FIELD APPROXIMATION}

In this section, we introduce a very simple mean-field approximation based on the slave rotors variables. This approximation is similar in spirit to the condensation of slave bosons in conventional slave boson mean-field theories. We illustrate this approximation on two examples: the Anderson impurity model and the Hubbard model. 


\section{A. Anderson impurity model}

The Anderson impurity model describes a local orbital hybridized to a conduction electron bath:

$$
H=H_{a t}+\sum_{k \sigma} \epsilon_{\mathbf{k}} c_{k \sigma}^{\dagger} c_{k \sigma}+V \sum_{k \sigma}\left(c_{k \sigma}^{\dagger} d_{\sigma}+\text { h.c. }\right)
$$

This Hamiltonian can be rewritten in terms of the slave rotor and auxiliary fermion variables:

$$
\begin{aligned}
H & =\frac{U}{2} \hat{L}^{2}+\epsilon_{0} \sum_{\sigma} f_{\sigma}^{\dagger} f_{\sigma}+\sum_{k \sigma} \epsilon_{\mathbf{k}} c_{k \sigma}^{\dagger} c_{k \sigma} \\
& +V \sum_{k \sigma}\left(c_{k \sigma}^{\dagger} f_{\sigma} e^{-i \theta}+\text { h.c. }\right)
\end{aligned}
$$

submitted to the constraint (4). The simplest possible approximation is to decouple the rotor and fermion variables, leading to two effective Hamiltonians:

$$
\begin{gathered}
H_{f}^{\mathrm{eff}}=\left(\epsilon_{0}-h\right) \sum_{\sigma} f_{\sigma}^{\dagger} f_{\sigma}+\sum_{k \sigma} \epsilon_{\mathbf{k}} c_{k \sigma}^{\dagger} c_{k \sigma} \\
+V_{\text {eff }} \sum_{k \sigma}\left(c_{k \sigma}^{\dagger} f_{\sigma}+\text { h.c. }\right) \\
H_{\theta}^{\mathrm{eff}}=\frac{U}{2} \hat{L}^{2}+h \hat{L}+K \cos \theta
\end{gathered}
$$

The parameters $V_{\text {eff }}, K$ and $h$ in these expressions are determined by the coupled self-consistent equations:

$$
\begin{aligned}
V_{\mathrm{eff}} & =V\langle\cos \theta\rangle_{\theta} \\
K & =V \sum_{k \sigma}\left\langle c_{k \sigma}^{\dagger} f_{\sigma}+f_{\sigma}^{\dagger} c_{k \sigma}\right\rangle_{f} \\
\langle\hat{L}\rangle_{\theta} & =N\left[n_{F}\left(\epsilon_{0}-h\right)-\frac{1}{2}\right]
\end{aligned}
$$

in which the averages are calculated with the effective Hamiltonians above.

Let us first examine the particle-hole symmetric case $\epsilon_{0}=0(Q=N / 2)$ in which the solution of (23) is $h=0$. The rotor sector is described by the effective Hamiltonian (20) corresponding to the Schrödinger equation:

$$
\left[-\frac{U}{2} \frac{\partial^{2}}{\partial \theta^{2}}+K \cos \theta\right] \Psi(\theta)=E \Psi(\theta)
$$

For $K=0$, the ground-state wave function is the state $|l=0\rangle$, uniform on $[0,2 \pi]$, corresponding to maximal phase fluctuations and thus to the absence of charge fluctuations. This is associated with the atomic limit, as explained above. As soon as the hybridization $V$ is nonzero, we shall see that $K \neq 0$. The wave function is then maximum $(K<0)$ for $\theta=0,2 \pi$, and $\langle\cos \theta\rangle$ acquires a non-zero expectation value. This corresponds to a non-zero effective hybridization $V_{\text {eff }}=V\langle\cos \theta\rangle$, so that the auxiliary fermion effective Hamiltonian is that of a resonant level model. This captures the physics of the Kondo effect, and the corresponding Kondo resonance at the Fermi level.

Even though $V$ is a singular perturbation on the atomic limit, its effect can be easily understood analytically in the present framework by treating the potential energy $K \cos \theta$ perturbatively. To first order in $K$, the groundstate wave function reads:

$$
\left|\Psi_{0}^{(1)}\right\rangle=|0\rangle+\sum_{\ell \neq 0} \frac{\langle\ell|K \cos \theta| 0\rangle}{E_{0}-E_{\ell}}|\ell\rangle
$$

with $\langle\theta \mid \ell\rangle=e^{i \ell \theta}$ and $E_{\ell}=U \ell^{2} / 2$. This yields:

$$
\begin{aligned}
\langle\cos \theta\rangle_{\theta} & =\left\langle\Psi_{0}^{(1)}|\cos \theta| \Psi_{0}^{(1)}\right\rangle \\
& =-2 K \sum_{\ell \neq 0} \frac{|\langle 0|\cos \theta| \ell\rangle|^{2}}{E_{\ell}}=-\frac{2 K}{U}
\end{aligned}
$$

Hence, using (21), one obtains $K=-U V_{\text {eff }} / 2 V$, which yields the following self-consistent equation for the effective hybridization $V_{\text {eff }}$ when substituted into (22):

$$
V_{\mathrm{eff}}=-\frac{2 V^{2}}{U} \sum_{k \sigma}\left\langle c_{k \sigma}^{\dagger} f_{\sigma}+f_{\sigma}^{\dagger} c_{k \sigma}\right\rangle_{f}
$$

The right-hand side of this equation is easily evaluated for the resonant level model (19). For simplicity, we consider a flat conduction band $\epsilon_{\mathbf{k}} \in[-\Lambda, \Lambda]$, and focus on the universal regime: $\Lambda \gg U \gg \Gamma$, with $\Gamma \equiv \pi V^{2} / 2 \Lambda$. To dominant order in $1 / \Lambda$, (27) reads:

$$
\begin{aligned}
1 & =-N \frac{2 V^{2}}{U \Lambda} \int_{-\Lambda}^{0} \mathrm{~d} \omega \frac{\omega}{\omega^{2}+\left(V_{\mathrm{eff}}^{2} \pi /(2 \Lambda)\right)^{2}} \\
& =N \frac{2 V^{2}}{U \Lambda} \ln \left(\frac{2 \Lambda^{2}}{\pi V_{\mathrm{eff}}^{2}}\right)
\end{aligned}
$$

This yields the following expression for $V_{\text {eff }}$ and for the width of the Kondo resonance when $\Lambda \gg U \gg \Gamma$ :

$$
\Gamma_{\mathrm{eff}} \equiv \frac{\pi V_{\mathrm{eff}}^{2}}{2 \Lambda}=\Lambda \exp \left(-\frac{\pi U}{4 N \Gamma}\right)
$$

This coincides with the exact expression [23]. The local orbital spectral function obtained from (14) reads:

$$
\rho_{d}(\omega)=Z \frac{\Gamma_{\mathrm{eff}} / \pi}{\omega^{2}+\Gamma_{\mathrm{eff}}^{2}}+\rho_{d}^{i n c}(\omega)
$$

The first term in this expression is the Kondo resonance, and carries a spectral weight $Z=\Gamma_{\text {eff }} / \Gamma=\langle\cos \theta\rangle_{\theta}^{2}$. It satisfies the Friedel sum-rule $\rho_{d}(\omega=0)=1 / \pi \Gamma$. Away from the particle-hole symmetric case $\left(\epsilon_{0} \neq 0\right)$, the location of the resonance is set by $\epsilon_{0}-h$, which is the renormalized impurity level familiar from conventional slaveboson theories. The rotor approximation does conserve total spectral weight, and therefore yields an incoherent contribution to the spectral function with a weight $1-Z$. This incoherent contribution is correctly centered around 
the atomic transitions, as explained above. However, the width of these Hubbard bands is incorrectly described by the simple approximation presented here, in which phase fluctuations are underestimated at short times. As a result, the Hubbard bands have a bandwidth of order $\Gamma_{\text {eff }}$ in this approximation (instead of the expected, and much broader width, of order $\Gamma$ ). We note however that conventional slave-boson approximations with a condensed boson neglect altogether the Hubbard bands at the saddle-point level, and therefore the present approximation, simplified as it may be, is preferable in this respect. An improved method for the treatment of phase degrees of freedom, leading to a much more accurate description of the Hubbard bands, has been discussed in previous publications [7, 8]. This method consists in a set of coupled integral equations for the Green's functions of the auxiliary fermion and of the slave rotor, in the spirit of the non-crossing approximation.

\section{B. Hubbard model}

\section{Slave rotor formulation}

In this section, we consider the Hubbard model:

$$
H=\sum_{i} H_{a t}(i)-\sum_{\mathrm{ij}, \sigma} t_{\mathrm{ij}} d_{\mathrm{i} \sigma}^{\dagger} d_{\mathrm{j} \sigma}
$$

which can be rewritten in terms of the rotor and auxiliary fermion variables as:

$$
H=\sum_{\mathrm{i} \sigma} \epsilon_{0} f_{\mathrm{i} \sigma}^{\dagger} f_{\mathrm{i} \sigma}+\frac{U}{2} \sum_{\mathrm{i}} \hat{L}_{\mathrm{i}}^{2}-\sum_{\mathrm{ij} \sigma} t_{\mathrm{ij}} f_{\mathrm{i} \sigma}^{\dagger} f_{\mathrm{j} \sigma} e^{i\left(\theta_{\mathrm{i}}-\theta_{\mathrm{j}}\right)}
$$

Note that, in this context, $-\epsilon_{0}=\mu$ is the chemical potential controlling the average density per site. Let us make a first approximation, which consists in decoupling the rotor and fermion variables on links (besides treating the constraint on average, as above), see 24 for a similar approach in the case of the t-J model. We then obtain two effective Hamiltonians:

$$
\begin{gathered}
H_{f}=-\sum_{\mathrm{ij} \sigma} t_{\mathrm{ij}}^{\mathrm{eff}} f_{\mathrm{i} \sigma}^{\dagger} f_{\mathrm{j} \sigma}+\left(\epsilon_{0}-h\right) \sum_{\mathrm{i} \sigma} f_{\mathrm{i} \sigma}^{\dagger} f_{\mathrm{i} \sigma} \\
H_{\theta}=-\sum_{i j} \mathcal{J}_{\mathrm{ij}}^{\mathrm{eff}} \cos \left(\theta_{i}-\theta_{j}\right)+\sum_{\mathrm{i}}\left(\frac{U}{2} \hat{L}_{i}^{2}+h \hat{L}_{i}\right)
\end{gathered}
$$

corresponding respectively to free fermionic spinons with an effective hopping $t_{\mathrm{ij}}^{\mathrm{eff}}$ and to a quantum XY-model for the phase variables with effective exchange constants $\mathcal{J}_{\text {ij }}^{\text {eff }}$. These effective parameters are determined by coupled self-consistent equations:

$$
t_{\mathrm{ij}}^{\text {eff }}=t_{\mathrm{ij}}\left\langle\cos \left(\theta_{i}-\theta_{j}\right)\right\rangle_{\theta}, \mathcal{J}_{\mathrm{ij}}^{\mathrm{eff}}=\sum_{\sigma} t_{\mathrm{ij}}\left\langle f_{\mathrm{i} \sigma}^{\dagger} f_{\mathrm{j} \sigma}\right\rangle_{f}
$$

in which the average values are calculated with the effective Hamiltonians above. In addition, the Lagrange multiplier $h$ is determined from the constraint equation:

$$
\langle\hat{L}\rangle_{\theta}=\sum_{\sigma}\left(\left\langle f_{\mathrm{i} \sigma}^{\dagger} f_{\mathrm{i} \sigma}\right\rangle_{f}-\frac{1}{2}\right)
$$

Let us emphasize that, in the decoupling leading to (3334), we have assumed that the average values $\left\langle f_{\mathrm{i} \sigma}^{\dagger} f_{\mathrm{j} \sigma}\right\rangle_{f}$ and $\left\langle\exp i\left(\theta_{i}-\theta_{j}\right)\right\rangle_{\theta}$ on a given bond are both real. In fact, one could look for more general classes of solutions in which both $\left\langle f_{\mathrm{i} \sigma}^{\dagger} f_{\mathrm{j} \sigma}-f_{\mathrm{j} \sigma}^{\dagger} f_{\mathrm{i} \sigma}\right\rangle_{f} \neq 0$ and $\left\langle\sin \left(\theta_{i}-\theta_{j}\right)\right\rangle_{\theta} \neq 0$. This would correspond to solutions with orbital currents around a plaquette, as proposed by several authors 25]. Spontaneous orbital currents are very naturally described using the slave rotor method, but will not be considered further in this paper, which aims at the general formalism.

\section{Simplest mean-field}

In the next section, we shall investigate some physical consequences of equations (333435) which approximate the Hubbard model by free spinons coupled selfconsistently to an XY-model for the phase degrees of freedom. We point out that the decoupling between fermion and rotor degrees of freedom can be viewed as a controlled approximation corresponding to a large- $\mathrm{N}$ limit of a multichannel model, as detailed in appendix $\mathrm{A}$

Here, in the same spirit as above, we consider a further simplification, which consists in treating the quantum XY model at the mean-field level. In this framework, the phase degrees of freedom is described by a mean-field Hamiltonian of independent sites:

$$
H_{\theta}^{\mathrm{MF}}=\sum_{\mathrm{i}}\left[\frac{U}{2} \hat{L}_{i}{ }^{2}+h \hat{L}_{i}+K \cos \theta_{i}\right]
$$

with $K=-2 \sum_{j} \mathcal{J}_{\mathrm{ij}}^{\text {eff }}\left\langle\cos \theta_{j}\right\rangle_{\theta}$. Combining this with (35) and calculating the average values with the free-fermion Hamiltonian (33), we finally obtain the following selfconsistency equations for the variational parameters $K$ and $h$ :

$$
\begin{aligned}
K & =2 N\langle\cos \theta\rangle \int \mathrm{d} \epsilon D(\epsilon) \epsilon n_{F}\left(Z \epsilon+\epsilon_{0}-h\right) \\
\langle\hat{L}\rangle & =N \int \mathrm{d} \epsilon D(\epsilon)\left[n_{F}\left(Z \epsilon+\epsilon_{0}-h\right)-\frac{1}{2}\right] \\
Z & \equiv\langle\cos \theta\rangle_{\theta}^{2}
\end{aligned}
$$

Finally, the relation between the chemical potential $-\epsilon_{0}$ and average number of particle per site and color $n$ is given by:

$$
n \equiv \frac{1}{N} \sum_{\sigma}\left\langle f_{\sigma}^{\dagger} f_{\sigma}\right\rangle=\int \mathrm{d} \epsilon D(\epsilon) n_{F}\left(Z \epsilon+\epsilon_{0}-h\right)
$$


In these expressions, $D(\epsilon) \equiv \int \frac{d^{d} k}{(2 \pi)^{d}} \delta\left(\epsilon-\epsilon_{\mathbf{k}}\right)$ is the density of states (d.o.s) of the band in the absence of interactions. The auxiliary fermion (quasiparticle) Green's function reads:

$$
G_{f}\left(\mathbf{k}, i \omega_{n}\right)^{-1}=i \omega_{n}-\epsilon_{0}+h-Z \epsilon_{\mathbf{k}}
$$

We recognize $Z$ as the quasiparticle weight, which also determines the quasiparticle mass enhancement $m^{\star} / m=$ $1 / Z$. These two quantities are related because of the simple single-site approximation made here.

At zero temperature, the number equation (40) implies that:

$$
h-\epsilon_{0}=Z \mu_{0}(n)
$$

in which $\mu_{0}$ is the chemical potential of the noninteracting system such that $\int_{-\infty}^{\mu_{0}} d \epsilon D(\epsilon)=n$. From (41), it is seen that the Fermi surface is located at $\epsilon_{\mathbf{k}}=\left(h-\epsilon_{0}\right) / Z$, and thus (42) implies that Luttinger theorem is satisfied. In fact, within this simple approximation in which the self-energy is independent of momentum, the Fermi surface is unchanged by interactions altogether. The equations for $K$ and $h$, at $T=0$ and for a given density, simplify into:

$$
\begin{aligned}
\langle\hat{L}\rangle & =N\left(n-\frac{1}{2}\right) \\
K & =2 N \bar{\epsilon}(n)\langle\cos \theta\rangle_{\theta}
\end{aligned}
$$

with $\bar{\epsilon}(n) \equiv \int_{-\infty}^{\mu_{0}(n)} d \epsilon D(\epsilon) \epsilon$ the average kinetic energy per electronic degree of freedom in the non-interacting model.

\section{Mott transition and orbital degeneracy}

We expect a Mott transition to occur at each commensurate filling $n=Q / N$ ( $Q$ being an integer). This is associated with the vanishing of $Z$, and therefore the above equations can be analyzed analytically close to the transition (where $Z$ is small) from a perturbative analysis in $K$, similar to the one performed in section IIIA for the Anderson model in the Kondo regime. The ground-state wave function of $H_{\theta}^{\mathrm{MF}}$ in the insulating phase $(Z=K=0)$ is $e^{i \ell_{n} \theta}$ with $\ell_{n}=N(n-1 / 2)$. First-order perturbation theory in $K$ yields:

$$
\begin{aligned}
\langle\cos \theta\rangle_{\theta} & =2 K \sum_{\ell \neq \ell_{n}} \frac{\left|\left\langle\Psi_{\ell}|\cos \theta| \Psi_{\ell_{n}}\right\rangle\right|^{2}}{E_{\ell_{n}}-E_{\ell}} \\
& =-\frac{2 U K}{U^{2}-4\left(U \ell_{n}+h\right)^{2}}+\mathcal{O}\left(K^{2}\right)
\end{aligned}
$$

Since $Z$ vanishes at the transition, but $\mu_{0}(n)$ is finite, it follows from (42) that $h=\epsilon_{0}$. For vanishing $Z$, the relation between $\epsilon_{0}$ and $n$ is identical to that of the atomic limit, Eq. (13) established in the previous section: $\ell_{n}=\operatorname{Int}\left[1 / 2-\epsilon_{0} / U\right]$ with $n=1 / 2+\ell_{n} / N$. Finally, combining (45) and (44), we obtain:

$$
U_{c}\left(\epsilon_{0}\right)^{2}-4\left[U_{c}\left(\epsilon_{0}\right) \ell_{n}+\epsilon_{0}\right]^{2}+4 N \bar{\epsilon}(n) U_{c}\left(\epsilon_{0}\right)=0
$$

In this expression, $\ell_{n}$ and $n$ should be viewed as depending on the chemical potential $\epsilon_{0}$ according to the relations just given. This expression determines the boundary $U_{c}\left(\epsilon_{0}\right)$ between the metallic and Mott insulating phase in the $\left(\epsilon_{0}, U\right)$ plane. It is depicted for the case $N=4$ (two orbitals with spin) in Fig. [3. The condition $\partial U_{c} / \partial \epsilon_{0}=0$

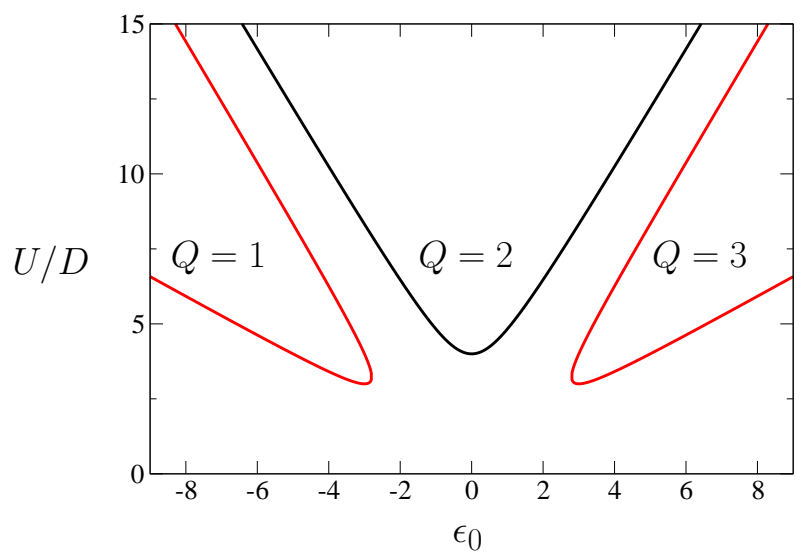

FIG. 3: Phase diagram for $N=4$ (two orbitals) at $T=$ 0 , as a function of the chemical potential $\epsilon_{0}=-\mu$ and the interaction strength $U / D$. The three lobes correspond to the Mott insulator phases associated with half-filling $(Q=2)$ and quarter-filling $(Q=1,3)$ respectively.

determines the tip of each insulating lobe, i.e the critical coupling $U_{c}(n)$ at which the insulating phase is entered as one increases $U$ for a fixed commensurate density $n$. Differentiating (46), it is seen that this happens for $\epsilon_{0}=-U l_{n}$, i.e precisely at the center of each step of the Coulomb staircase. The critical coupling thus reads:

$$
U_{c}(n)=4 N|\bar{\epsilon}(n)|
$$

The phase diagram in the $(n, U)$ plane is depicted in Fig. 4 for a flat d.o.s of half-width $D$, in which case $U_{c}=4 N D n(1-n)$. We see that the critical coupling is biggest at half-filling $n=1 / 2(Q=N / 2)$, which is expected since orbital fluctuations are largest in this case. This conclusion may depend on the precise shape of the d.o.s however (and in particular may not hold for densities of states such that $D(-\epsilon) \neq D(\epsilon)$ ). The critical coupling increases linearly with orbital degeneracy $N$. In fact, an analysis of the DMFT equations for large orbital degeneracy was made in Ref. 11], and the exact behavior of the critical coupling at leading order in $N$ found there is correctly reproduced by the simple mean-field detailed here. It is also instructive to compare the present results with that of the multi-orbital Gutzwiller approximation [26], which reads: $U_{c}^{\mathrm{GA}}=4(N+2)|\bar{\epsilon}(n)|$. Our 


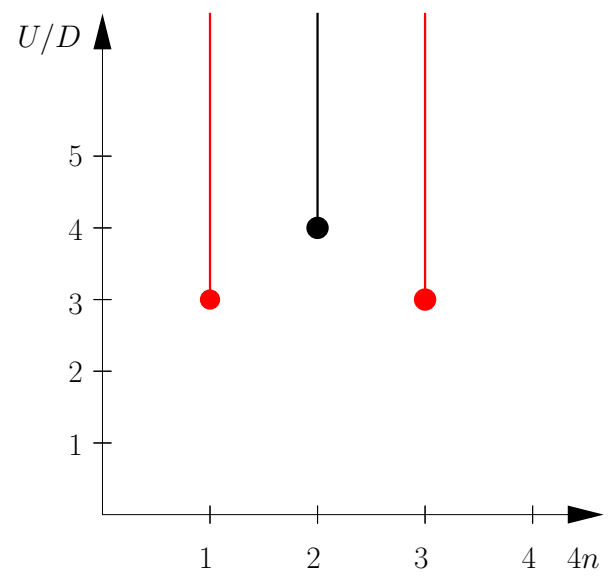

FIG. 4: Phase diagram in the $(n, U)$ plane. The Mott insulator lobes collapse to lines at commensurate fillings, when $U$ is larger than $U_{c}(n)$ (shown as dots).

expression has the same behavior at large $N$, but yields in general a smaller critical coupling: $U_{c}=U_{c}^{\mathrm{GA}} N /(N+2)$. For small orbital degeneracies, we believe (on the basis of, e.g, DMFT results) the Gutzwiller expression of $U_{c}$ to be quantitatively more accurate.

The slave-rotor mean field equations are easily solved numerically by determining iteratively the parameters $h$ and $K$. At each iteration, the spectrum of the singlerotor Schrödinger equations is computed (using e.g a decomposition on the atomic basis states $\left.e^{i \ell \theta}\right)$. In Fig. 5 the ground-state wave function $\Psi_{0}(\theta)$ is displayed for several values of $U$ at half-filling. The curves nicely illustrate

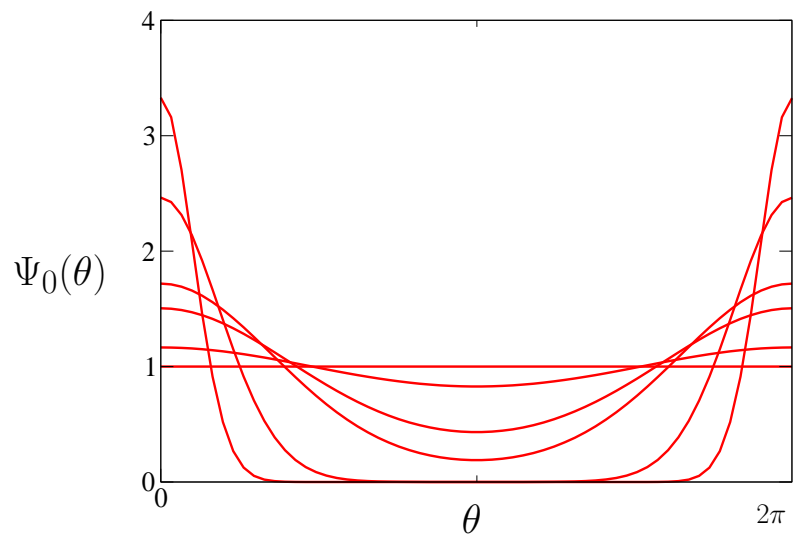

FIG. 5: Rotor ground state wave function $\Psi_{0}(\theta)$ with values of the local interaction ranging from $U / U_{c}=0.01$ (peaked curve) to $U / U_{c}=1$ at the Mott transition (flat curve).

how one goes from the insulator (in which case there are little charge fluctuations, and maximal phase fluctuations so that the wave-function is delocalized over all $\theta$ values) to the metal (in which case charge fluctuations become large at small $U$, and the wave-function is peaked such as to limit phase fluctuations). The corresponding quasiparticle weight is displayed in Fig. 6] as a function of $U / U_{c}$. The simple slave-rotor mean-field is compared to the DMFT result and to the Gutzwiller approximation (GA). It is seen that, close to the transition, the slaverotor mean field reproduces more accurately the DMFT answer than the GA. It is not very accurate at weakcoupling however (even though $Z$ correctly goes to $Z=1$ at $U=0$, it has an incorrect small- $U$ expansion). In fact, it is a quite general feature of this slave-rotor mean field that the method is more accurate in strongly correlated regimes.

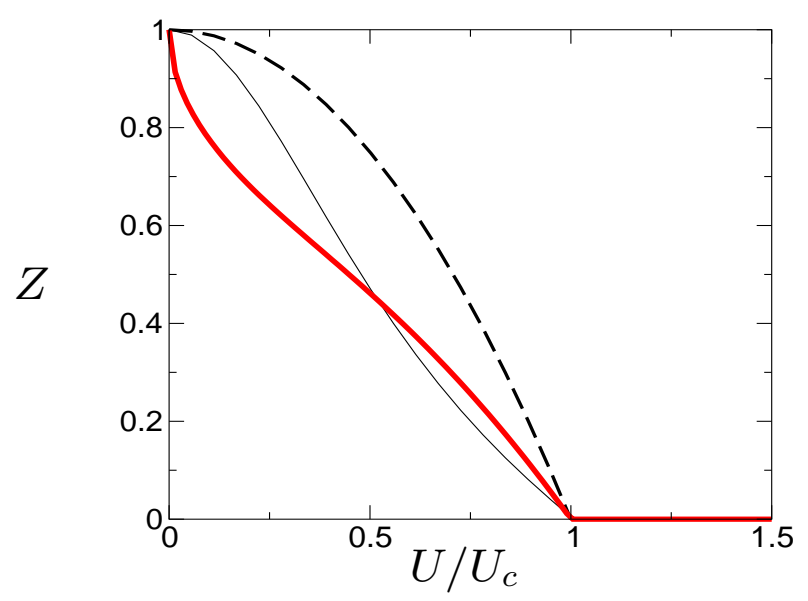

FIG. 6: Quasi-particle weight $Z$ as a function of $U / U_{c}$ at $T=0$; DMFT calculation (thin line), rotor mean-field theory (thick line) and Gutzwiller approximation (broken line).

In Fig. 7 we plot the number of particles as a function of the chemical potential for $N=4$. The value of $U$ has been chosen to be bigger than the critical couplings yielding an insulating state, for any commensurate filling. The curve illustrates the plateaus found at each commensurate filling, the central one (half-filling) being narrower (compare to Fig. 31). The effective mass enhancement $(=1 / Z)$ is also plotted in Fig. 8 as a function of chemical potential for a smaller value of $U$, such that a metallic phase is found at any filling. The curves illustrates how a largest effective mass enhancement is found at low and high fillings $n=1 / 4,3 / 4$, and a comparatively smaller close to half-filling $n=1 / 2$ (again, this conclusion depends on the shape of the d.o.s).

The description of the Mott transition obtained within this simplest mean-field has many common features with the Brinkman-Rice (BR) [27] one. Indeed, the effective mass diverges at the transition and the quasi-particle residue vanishes $\left(Z \sim 1-U / U_{c}\right)$ as in BR. There is one significant difference however, which is that in the present description, the optical gap $\Delta$ of the insulator does not coincide with the chemical potential jump $\Delta \mu=-\Delta \epsilon_{0}=\mu\left(n+0^{+}\right)-\mu\left(n-0^{+}\right)$for infinitesimal doping away from a commensurate filling. Indeed, within this simple mean-field, the spectral function of the insu- 


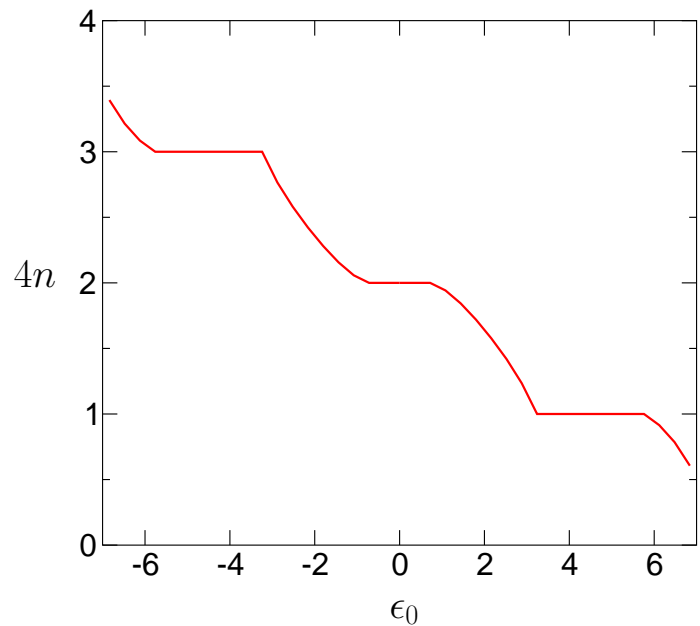

FIG. 7: Total occupancy $Q=4 n$ as a function of $\epsilon_{0}$ for a value $U=4.5$ larger than all critical interactions $U_{c}(n)$, in the two orbital case $(N=4)$. The Mott insulators are seen here as charge plateaus.

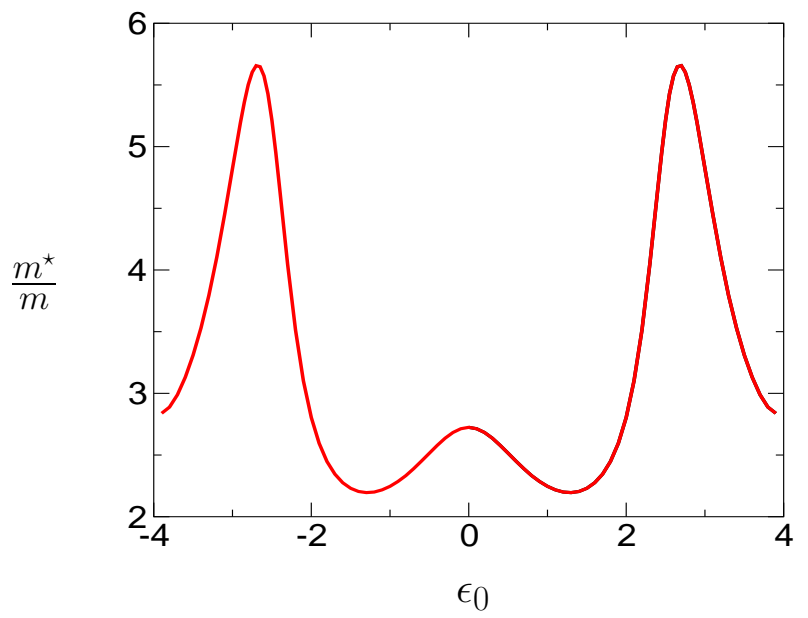

FIG. 8: Effective mass $m^{\star} / m$ for $U=2.5$ below all Mott transitions, in the two orbital case $(N=4)$.

lator is identical to that of the atomic limit (not surprisingly, the simple mean-field with only two variational parameters describes the charge fluctuations in the insulator in an oversimplified manner). As a result, the optical gap simply reads

$$
\Delta=U
$$

in our approach, and is therefore not critical at the Mott transition. In contrast, the chemical potential jump vanishes continuously at $U_{c}$. Indeed, solving (46) for $\epsilon_{0}$ yields:

$$
\Delta \mu=U \sqrt{1-\frac{U_{c}}{U}}
$$

These features are very similar to those obtained within dynamical mean-field theory [2]. This is not surprising, since the single-site mean field approximation to the XYmodel indeed becomes exact in the limit of infinite coordination of the lattice. Note however that this is not the case of the approximation (33)-(35) which consists in decoupling the rotor and fermion variables (see section IV). Within DMFT, the quasiparticle weight vanishes at a Brinkman-Rice like critical point $U_{c 2}$ while the optical gap of the insulator vanishes at a Hubbard-like critical point $U_{c 1}$. As a result, the strongly correlated metal close to the transition displays a clear separation of energy scales: the quasiparticle coherence scale $\epsilon_{F}^{\star} \sim Z D$ being much smaller than the ("preformed") gap of the insulator $\Delta$. The simple mean-field of this section is in a sense a somewhat extreme simplification of this picture, in which $U_{c 2}=U_{c}$ and $U_{c 1}$ is sent to $U_{c 1}=0$ (this is consistent with the known fact [1] that $U_{c 2} \propto N$ while $U_{c 1} \propto \sqrt{N}$, and that the simple mean-field becomes more accurate for large- $N$ ).

\section{INCLUDING SPATIAL CORRELATIONS AND PHASE FLUCTUATIONS}

In this section, we go beyond the single-site meanfield approximation, and investigate the physical consequences of the approximate description of the Hubbard model introduced in Sec. IIIB 1 This description, summarized by Eqs. (33) 35), consists in a free fermion model $H_{f}$ coupled self-consistently to a quantum XY-model $H_{\theta}$ for the phase degrees of freedom.

\section{A. General considerations}

Let us first emphasize some general aspects of this description, before turning to explicit calculations. The Hamiltonian for the phase degrees of freedom has two possible phases: a disordered phase without long-range phase order, and a long-range ordered phase. At zerotemperature, one expects a quantum phase transition from the ordered phase to the disordered phase as the ratio $U / \mathcal{J}_{\mathrm{ij}}^{\text {eff }}$ is increased. Since the Green's function of the physical electrons read, within this approximation:

$$
G_{i j}^{d}\left(\tau-\tau^{\prime}\right)=G_{i j}^{f}\left(\tau-\tau^{\prime}\right)\left\langle e^{i\left[\theta_{i}(\tau)-\theta_{j}\left(\tau^{\prime}\right)\right]}\right\rangle_{\theta}
$$

it is seen that the quasiparticle weight $Z$, associated with the limit of large-distance and large time separation (low frequency), is given by:

$$
Z=\left\langle\cos \theta_{i}\right\rangle_{\theta}^{2}
$$

Thus, the phase with long-range order for the rotors corresponds to the metal $(Z \neq 0)$, while the disordered phase correspond to the Mott insulator $(Z=0)$. Obviously, the description of the Mott metal-insulator transition that follows is closely analogous to that of the 
superfluid-Mott insulator transition in the bosonic Hubbard model [28, 29]. Two remarks about this description of the metal and of the insulator are in order. First, it is of course unphysical to think of a metal as having longrange phase coherence. Naturally, this is only true of the saddle-point approximation in which the rotors and spinon degrees of freedom are entirely decoupled. Fluctuations will induce interactions between these degrees of freedom, restore inelastic scattering and thus destroy phase coherence. The absence of inelastic scattering at the saddle-point level is a well-known feature of slaveboson theories. Note futhermore that despite the ordering of the rotors, the metallic phase becomes a superconductor only when $\left\langle f_{i \uparrow}^{\dagger} f_{j \downarrow}^{\dagger}\right\rangle$ is also non-zero (i.e when there is spinon pairing). Second, the insulator envisioned here is a non-magnetic insulator without any spin or translational symmetry breaking, i.e a spin-liquid. Even in the disordered phase, $\left\langle\cos \left(\theta_{i}-\theta_{j}\right)\right\rangle$ on a given bond (e.g nearest-neighbour) is non-zero (it corresponds to the energy density of the XY model). Therefore $t_{\mathrm{ij}}^{\mathrm{eff}} \neq 0$ in the insulating phase, so that the spinons have a Fermi surface (with Luttinger volume). This also implies that $t_{\mathrm{ij}}^{\mathrm{eff}}$ remains finite through the Mott transition and therefore that the effective mass does not diverge, despite the fact that $Z \rightarrow 0$. These last remarks apply to any finite dimension, but of course not to $d=\infty$. In this limit, the single-site mean field of the previous section applies and $\left\langle\exp \left(\theta_{i}-\theta_{j}\right)\right\rangle=\langle\cos \theta\rangle^{2}=Z$. Finally, we emphasize that the non-magnetic nature of the insulator is of course associated with the fact that the rotor degrees of freedom are associated with the charge and are not appropriate to properly describe spin ordering. Therefore, they are better suited to lattices with strong frustration (or models with large orbital degeneracy) in which a spin-liquid insulator is a realistic possibility. Finally, because long-range order for the rotors corresponds to breaking a continuous $\mathrm{O}(2)$ symmetry, a Goldstone mode will be present in the ordered (metallic) phase. This mode is present in any finite dimension, but disappears in the $d=\infty$ limit. It corresponds to the zero-sound mode of the metal. As we shall see, these long-wavelength modes play an important role: they change the low-energy description of the transition as compared to the $d=\infty$ (DMFT) limit. As a result, the separation of energy scales does not apply in a strict sense (the "preformed" gap found within DMFT is filled up with spectral weight coming from these low-energy modes). As we shall see however, this spectral weight remains small in high dimensions (including $d=3$ ), so that an approximate separation of scales still applies.

\section{B. Sigma-model representation: saddle-point equations in the spherical limit}

In order to perform explicit calculations with the quantum rotor Hamiltonian (34), we shall use an approximation that has proven successful in the context of quantum impurity models with slave rotors 7,8$]$. It consists in replacing the quantum rotor $\exp \left(i \theta_{i}\right)$ by a complex bosonic field $X_{i}(\tau)$ and to treat the constraint $\left|X_{i}\right|^{2}=1$ on average. Alternatively, this can be viewed as extending the $\mathrm{O}(2)$ symmetry to $\mathrm{O}(\mathrm{M})$ and taking the large-M (spherical) limit. This is a well known approximation to nonlinear sigma models [29], which preserves many qualitative features of the quantum phase transition. For details of the formalism in the slave rotor context, see Ref. 7]. In the following, we focus on the half-filled case (since we are mainly interested in the Mott transition), with a particle-hole symmetric d.o.s $D(\epsilon)$, so that we can set $\epsilon_{0}=h=0$

The spinon and rotor (now X-field) Green's function read [39]:

$$
\begin{array}{r}
G_{f}\left(\mathbf{k}, i \omega_{n}\right)^{-1}=i \omega_{n}-Q_{f} \epsilon_{\mathbf{k}} \\
G_{X}\left(\mathbf{k}, i \nu_{n}\right)^{-1}=\frac{\nu_{n}^{2}}{U}+\lambda+Q_{X} \epsilon_{\mathbf{k}}
\end{array}
$$

In these expressions, $\omega_{n}$ and $\nu_{n}$ are respectively, fermionic and bosonic Matsubara frequencies, $\lambda$ is a Lagrange multiplier associated with the constraint $\left\langle|X|^{2}\right\rangle=$ 1, while $Q_{f}$ and $Q_{X}$ are the self-consistent parameters entering the effective spinon hopping and XY coupling constants: $Q_{f}=\left\langle\cos \left(\theta_{i}-\theta_{j}\right)\right\rangle=\left\langle X_{i} X_{j}^{\star}\right\rangle, Q_{X}=$ $\left\langle\sum_{\sigma} f_{\mathrm{i} \sigma}^{\dagger} f_{\mathrm{j} \sigma}\right\rangle$. The self-consistent equations which determine $\lambda, Q_{f}$ and $Q_{X}$ read:

$$
\begin{aligned}
1 & =\int_{-D}^{D} \mathrm{~d} \epsilon D(\epsilon) \frac{1}{\beta} \sum_{n} \frac{1}{\nu_{n}^{2} / U+\lambda+Q_{X} \epsilon} \\
D Q_{f} & =-\int_{-D}^{D} \mathrm{~d} \epsilon D(\epsilon) \epsilon \frac{1}{\beta} \sum_{n} \frac{1}{\nu_{n}^{2} / U+\lambda+Q_{X} \epsilon}(55) \\
D Q_{X} & =-2 \int_{-D}^{D} \mathrm{~d} \epsilon D(\epsilon) \epsilon n_{F}\left(Q_{f} \epsilon\right)
\end{aligned}
$$

These expressions have been written here for a simple tight-binding band with nearest-neighbor hopping $t_{i j}=t$ on a $d$-dimensional cubic lattice $\left(\epsilon_{\mathbf{k}}=-2 t \sum_{\alpha=1}^{d} \cos k_{\alpha}\right)$. As above, $D(\epsilon)$ denotes the band d.o.s, and $D=2 d t$ is the half-bandwidth. For simplicity, we have set the orbital degeneracy to $N=2$ in these equations.

\section{The Mott transition: Mott-Hubbard meets Brinkman-Rice}

In this section, we investigate the solution of these equations at zero-temperature. This leads to a description of the finite-dimensional Mott transition that we analyze in detail. 


\section{The insulating phase}

Lets us note first that Eq. (56) readily determines $Q_{X}$ at $T=0$ :

$$
\left.D Q_{X}\right|_{T=0}=-2 \int_{-D}^{0} D(\epsilon) \epsilon d \epsilon \equiv 2|\bar{\epsilon}|
$$

From the form (53) of the X-field Green's function, one sees that the bosonic spectrum has a gap as long as $\lambda-$ $Q_{X} D=\lambda-2|\bar{\epsilon}|>0$. In this case, there is no longrange order for the phase degree of freedom, and this corresponds to the insulating phase. The insulating gap reads:

$$
\Delta_{g}=2 \sqrt{U\left(\lambda-Q_{X} D\right)}
$$

and we can rewrite Eqs. (5455) as selfconsistent equations for the gap $\Delta_{g}$ and the renormalization of the spinon hopping $Q_{f}$. This reads, at $T=0$ :

$$
\begin{aligned}
1 & =\int_{-D}^{D} \mathrm{~d} \epsilon D(\epsilon) \frac{U}{\sqrt{\Delta_{g}^{2}+8 U|\bar{\epsilon}|(1+\epsilon / D)}} \\
Q_{f} & =-\int_{-D}^{D} \mathrm{~d} \epsilon D(\epsilon) \frac{\epsilon}{D} \frac{U}{\sqrt{\Delta_{g}^{2}+8 U|\bar{\epsilon}|(1+\epsilon / D)}}
\end{aligned}
$$

These equations are valid in the insulating phase, when $\Delta_{g}>0$. The gap vanishes at a critical coupling $U_{c}$ obtained by setting $\Delta_{g}=0$ in (59):

$$
\frac{U_{c}}{U_{c}^{\infty}}=\left[\int_{-D}^{D} \mathrm{~d} \epsilon \frac{D(\epsilon)}{\sqrt{1+\epsilon / D}}\right]^{-2}
$$

In this expression, $U_{c}^{\infty}=8|\bar{\epsilon}|$ is the critical coupling corresponding to the $d=\infty$ limit, in agreement with expression (47) of the previous section (with $N=2$ ). Note that, in the $d \rightarrow \infty$ limit, one must scale the hopping as $t=t^{\star} / \sqrt{d}$, so that $D=2 d t \propto \sqrt{d} \rightarrow \infty$ and the r.h.s of (61) goes to unity. The integral in (61) is smaller than unity in general, so that $U_{c}$ decreases as dimensionality is reduced. We also note that in one dimension, this integral has a logarithmic singularity at band edge, since $\pi D(\epsilon)=D / \sqrt{D^{2}-\epsilon^{2}}$, so that Eq. (61) yields $U_{c}^{d=1}=0$, which is indeed the exact result for a half-filled Hubbard model with $N=2$ [30] (see however [40]).

Substracting Eq. (59) from the same equation with $\Delta_{g}=0$ (which defines $U_{c}$ ), one obtains:

$$
\begin{aligned}
& \sqrt{\frac{U_{c}^{\infty}}{U_{c}}}-\sqrt{\frac{U_{c}^{\infty}}{U}}= \\
& \int_{-D}^{D} \mathrm{~d} \epsilon D(\epsilon)\left[\frac{1}{\sqrt{1+\epsilon / D}}-\frac{1}{\sqrt{\Delta_{g}^{2} /\left(U U_{c}^{\infty}\right)+1+\epsilon / D}}\right]
\end{aligned}
$$

The expansion of this expression for small $\Delta_{g}$ depends on dimensionality. For $d>3$, the integral $\int d \epsilon D(\epsilon)(1+$ $\epsilon / D)^{-3 / 2}$ is convergent at band edge $\epsilon=-D$, recalling that $D(\epsilon) \sim(D+\epsilon)^{d / 2-1}$ near the bottom of the band. In contrast, the small- $\Delta_{g}$ expansion is singular for $d<3$. This analysis finally leads to the following behavior of the gap close to the critical point:

$$
\begin{aligned}
\Delta_{g} / U_{c} & \propto \frac{U}{U_{c}}-1 \text { for } d>3 \\
& \propto\left(\frac{U}{U_{c}}-1\right)^{1 /(d-1)} \text { for } d<3
\end{aligned}
$$

Hence we find that the exponent changes from its meanfield value $1 / 2$ for $d>3$ (as found e.g in the single-site mean-field of the previous section and the Gutzwiller approximation) to a non- mean field exponent for $1<d<3$. Therefore, $d=3$ corresponds to the upper critical dimension in this description of the Mott transition (logarithmic corrections are found in that case). Below $d=3$, the exponent $1 /(d-1)$ corresponds to that of the large- $M$ limit of the quantum $O(M)$ model in $d$-dimensions, i.e to that of the $d+1$-dimensional classical model. Had we kept $O(2)$ quantum rotors, we would have found $\Delta_{g} \sim\left(U / U_{c}-1\right)^{z \nu}$ with $z=1$ and $\nu$ the correlationlength exponent of the $d+1$-dimensional classical XY model, as in the case of the superfluid-insulator transition of the Bose Hubbard model [28].

\section{The metallic phase}

For $U<U_{c}$, the gap closes and one enters the metallic phase. In this regime, the constraint equation $\left\langle|X|^{2}\right\rangle=$ 1 can only be satisfied by a Bose condensation of the $\mathrm{X}$-field. As in studies of quantum magnetism based on Schwinger bosons [31], Bose condensation in the spherical limit corresponds to the phase with long-range order for the rotors. In this phase, the constraint equation (54) has to be rewritten by isolating the $\mathbf{k}=0$ mode in the Brillouin zone. The Lagrange multiplier $\lambda$ sticks to the value $\lambda=Q_{X} D=2|\bar{\epsilon}|$ in this phase. The full X-field Green's function thus reads at $T=0$ :

$$
G_{X}(\mathbf{k}, i \nu)=Z \delta(\nu) \delta(\mathbf{k})+\frac{1}{\nu^{2} / U+2|\bar{\epsilon}|\left(1+\epsilon_{\mathbf{k}} / D\right)}
$$

The condensation amplitude $Z=\langle X\rangle^{2}$ is determined from the constraint $\left\langle|X|^{2}\right\rangle=\sum_{\mathbf{k}} G_{X}(\mathbf{k}, \tau=0)=1$ :

$$
1=Z+\int_{-D}^{D} \mathrm{~d} \epsilon D(\epsilon) \sqrt{\frac{U}{8 \bar{\epsilon}(1+\epsilon / D)}}
$$

which simply reads, using (61):

$$
Z=1-\sqrt{\frac{U}{U_{c}}}
$$

This expression vanishes linearly, $Z \sim\left(U_{c}-U\right) / 2 U_{c}$, at the critical point for all dimensions $d>1$. The fact that 
there is no change of critical behavior for $Z$ at $d=3$, in contrast to the gap, is due to the use of the spherical approximation. Had we kept $\mathrm{O}(2)$ rotors, we would find $Z \sim\left(1-U / U_{c}\right)^{\nu(d-1+\eta)}$, with $\nu$ and $\eta$ the critical exponents of the $d+1$-dimensional classical XY model. In the spherical approximation $\eta=0$ and $\nu=1 /(d-1)$ so that $Z \sim\left(1-U / U_{c}\right)$ also below $d=3$.

Hence, we have found that the quasiparticle weight and insulating gap vanish at a unique critical coupling. As shown below, the gap $\Delta_{g}$ given by (59) is the gap in the single particle spectral density of the insulator. It also coincides with the chemical potential jump $\Delta \mu$ when the present approach is extended away from half-filling. Hence, in this finite dimensional description of the Mott transition, we find a unique critical point corresponding both to Brinkman-Rice physics 27] (vanishing of $Z$ ) and to Mott-Hubbard physics [32] (gap opening). This is in strong contrast to the $d=\infty$ single-site mean field investigated in the previous section, and to the DMFT picture 2]. Below, we show that this is due to long wavelength collective modes filling in the preformed gap, and investigate in detail how the previous picture is recovered in the (singular) $d=\infty$ limit.

The equation (55) for the renormalization $Q_{f}$ of the effective hopping must be rewritten in the metallic phase to take into account the Bose condensed fraction. At $T=0$, it reads:

$$
Q_{f}=\frac{m}{m^{\star}}=Z-\sqrt{\frac{U}{U_{c}^{\infty}}} \int_{-D}^{D} \mathrm{~d} \epsilon D(\epsilon) \frac{\epsilon / D}{\sqrt{1+\epsilon / D}}
$$

This expression makes very clear that the effective mass remains finite at the critical point, while $Z \rightarrow 0$ (note that the integral in the r.h.s of (67) is negative so that $Q_{f} \geq Z$ ). In the $d \rightarrow \infty$ limit, one recovers $m / m^{\star}=Z$, since $D \propto \sqrt{d} \rightarrow \infty$. This calculation can be extended to the weakly doped Mott insulator at large $U$ and hole density $\delta$, with the result:

$$
\frac{m^{\star}}{m} \sim \frac{1}{t / U+\delta} \sim \frac{1}{J / t+\delta}
$$

Hence, the present theory correctly captures the magnetic exchange energy $J \propto t^{2} / U$, which quenches out the spin entropy (due to spinon degrees of freedom) in the insulator and hence prevents the effective mass from diverging at the Mott transition. This is expected from the fact that the spinons form a dispersive band in the insulating state and thus have an entropy depending linearly on $T$ at low temperature. These findings are entirely similar to the slave bosons mean-field theories of the $t-J$ model [33]. Fig. 9]and Fig. [10 illustrate graphically the physical quantities characterizing the Mott transition which we discussed previously.

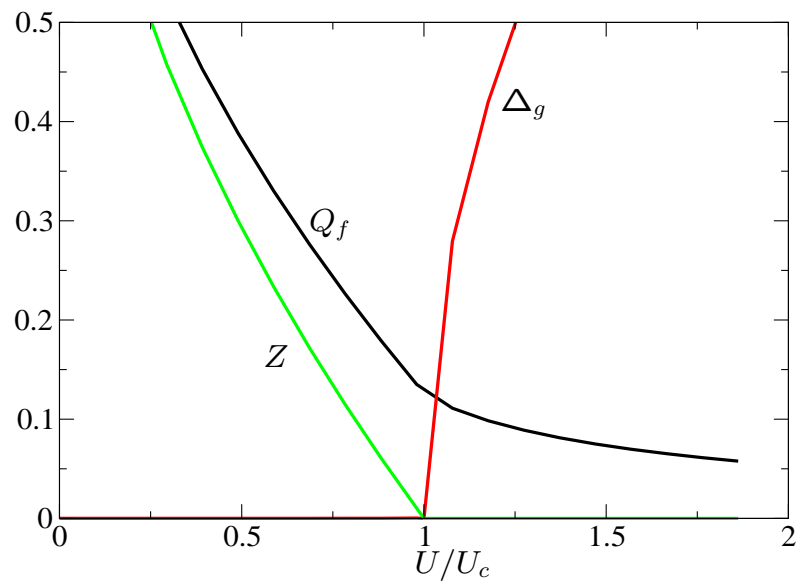

FIG. 9: Plot of the quasiparticle weight $Z$, the effective mass renormalization $Q_{f}=\mathrm{m} / \mathrm{m}^{\star}$ and the Mott gap $\Delta_{g}$ as a function of $U / U_{c}$ across the Mott transition in the threedimensional case.

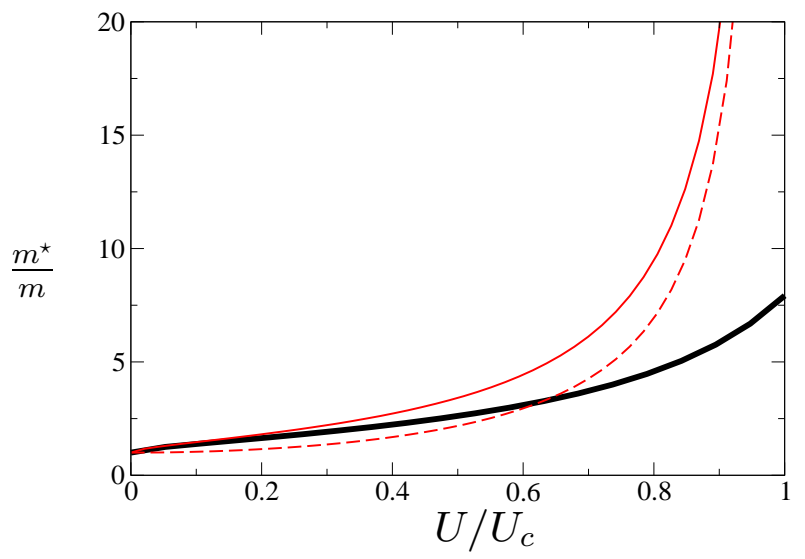

FIG. 10: Effective mass $m^{\star} / m=1 / Q$ provided by the meanfield equations (54 56) for $d=3$ (bold line) and $d=\infty$ (thin line). For comparison, a DMFT-IPT calculation (dashed line) is also presented.

\section{Spectral functions and collective modes: what are Hubbard bands made of.}

The Green's function of the physical electron in the approximation of decoupled spinons and rotors is given by (50) as: $G_{i j}^{d}(\tau)=G_{i j}^{f}(\tau) G_{i j}^{X}(\tau)$. Using (64) for the $\mathrm{X}$-field Green's function, this leads to:

$$
G_{d}(\mathbf{k}, i \omega)=\frac{Z}{i \omega-Q_{f} \epsilon_{\mathbf{k}}}+G_{d}^{\text {inc. }}(\mathbf{k}, i \omega)
$$

This expression is valid in the metallic phase. In Fig. 111 we display the $\mathbf{k}$-integrated (local) spectral function $\sum_{\mathbf{k}} \rho_{d}(\mathbf{k}, \omega)$, as the Mott transition is approached, in the three-dimensional case. The first term in (69) cor- 


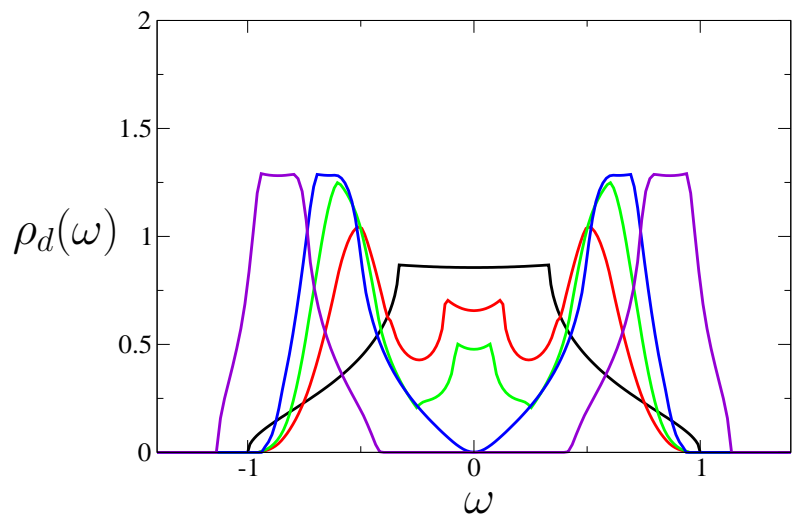

FIG. 11: Zero temperature local density of states across the Mott transition for a three-dimensional cubic lattice, with $U=0, U_{c} / 2, U_{c}, 3 U c / 2($ for $D=1)$.

responds to the coherent quasi-particle. When summed over $\mathbf{k}$, the quasiparticle contribution to the local spectral function yields a peak $Z / Q_{f} D\left(\omega / Q_{f}\right)$. The spectral weight of this peak is $Z$, its width is of order $Q_{f} D$ and its height is $Z D(0) / Q_{f}$. Hence, its height goes to zero at the transition, while its width is reduced but remains finite (Fig. 111). In the $d=\infty$ limit (where $Z=Q_{f}$ ) the zero-frequency density of states is pinned at its noninteracting value, as known from the Brinkman-Rice picture and the dynamical mean-field theory: only in infinite dimension does the quasiparticle peak disappear by narrowing down instead of collapsing.

The incoherent contribution $G_{d}^{\text {inc }}$ comes from the convolution of the free spinon Green's function with the non-condensed contribution to the rotor Green's function, $G_{X}^{\text {inc }}=1 /\left[\nu^{2} / U+2|\bar{\epsilon}|\left(1+\epsilon_{\mathbf{k}} / D\right)\right]$, which is the second term in (64). The latter corresponds to bosonic collective modes dispersing according to:

$$
\omega_{X}(\mathbf{k})= \pm \frac{\sqrt{U U_{c}^{\infty}}}{2} \sqrt{1+\frac{\epsilon_{\mathbf{k}}}{D}}
$$

The incoherent contribution corresponds to the Hubbard band, which are well developed in the correlated metal, as also predicted by DMFT. Note that the dispersing branch of bosonic excitations is centered around $\pm \sqrt{U U_{c}^{\infty}} / 2$. Hence a measure of the typical energy scale associated with the distance between the two Hubbard bands is:

$$
\Delta=\sqrt{U U_{c}^{\infty}}
$$

However, a key point is that this branch of collective modes extends to arbitrary low frequency where it becomes the Goldstone mode of the broken symmetry. In the small momentum limit, the dispersion relation (70) reads:

$$
\omega_{X}(\mathbf{k}) \sim \sqrt{\frac{U U_{c}^{\infty}}{8 d}}|\mathbf{k}|(\mathbf{k} \rightarrow 0)
$$

The corresponding density of states behaves as $\rho_{X}(\omega) \sim$ $\omega^{d-1}$. These long wavelength excitations are responsible for tails of the Hubbard bands, extending down to low- frequency. This low-energy spectral weight due to collective modes is the origin of the continuous closure of the Mott gap at the Brinkman-Rice transition. In other words, the Hubbard bands are made of two kinds of contributions. The main part of their spectral weight is associated with bosonic modes whose momentum is not small, so that $\omega_{X}$ is finite (of the order of $\Delta$ ). In addition, the small weight in the $\left(\sim \omega^{d-1}\right)$ tails at low-energy is associated with the $\mathbf{k} \simeq 0$ collective modes. In the Mott insulator, all the bosonic modes are gapped, but the bottom of the bosonic density of states has $\left(\omega-\Delta_{g}\right)^{d-1}$ tails which contribute to the tails of the Hubbard bands, filling in the energy range between $\Delta_{g}$ and $\Delta$.

It is natural to interpret the bosonic collective modes as the zero-sound mode of the metal. Indeed, these modes have been discussed previously by Castellani et al. [33], in their study of fluctuations around the saddle-point of conventional slave boson approaches. These authors pointed out that the Mott transition is associated with the softening of this collective mode, as also found here. In the present approach, the collective modes appear on the same footing than the quasiparticles.

\section{The $d=\infty$ limit, separation of energy scales and "preformed" Mott gap}

It is instructive to understand more precisely what happens as the dimensionality is increased. As clear from the previous discussion, the $d=\infty$ limit is singular in at least this respect that the long-wavelength collective modes are absent. Indeed the sound velocity in (72) vanishes in this limit. In fact the bosonic modes no longer have a dispersion: $\mathbf{k}$-dependence disappears from the dispersion relation (70) since $D$ must be scaled as $D \propto \sqrt{d}$. The bosonic spectral functions thus has two poles on top of the condensed fraction, which leads, after performing the convolutions, to the following simple form of the physical k-summed local spectral density (using also that $Q_{f}=Z$ in this limit):

$\rho_{d}^{d=\infty}(\omega)=D\left(\frac{\omega}{Z}\right)+\frac{1-Z}{2}\left[D\left(\frac{\omega-\Delta}{Z}\right)+D\left(\frac{\omega+\Delta}{Z}\right)\right]$

In this expression, $\Delta$ is given by (171) and corresponds to the typical separation between the Hubbard bands. It is sometimes referred in the framework of DMFT as the "preformed gap" in the metallic state (i.e the Hubbard bands are well separated from the central quasiparticle peak). $\Delta$ does not vanish at the Brinkman-Rice point and beyond this coupling the insulator sets in with a finite gap $\Delta$. Note that, in the present approximation where spinons and rotors have been decoupled, one simply has $\Delta=U$ in the insulator (as found also in (48)) and that, accordingly, the Hubbard bands in (73) have 
vanishing width close to the transition. This pathological result can be improved by including dynamical fluctuations of the auxiliary particles, as shown in [7]. Despite these oversimplifications, the present approach does retain the main qualitative feature of DMFT, namely the separation of energy scales at the Mott transition.

In Fig. 12 we show how the large-d limit is approached by plotting the local spectral density right at the critical coupling $U=U_{c}$, for increasing dimensionality. This plot clearly reveals the two-components building up the Hubbard bands, with the main part of the spectral weight centered around the "preformed" gap $\Delta$ and tails extending down to low-frequency (down to the true gap $\Delta_{g}$ in the insulator), associated with the long-wavelength collective mode. The inset demonstrates that as dimensionality increases, the spectral weight in the tails becomes smaller, so that an approximate separation of energy scales holds (and in fact already holds to a good approximation in $d=3$, while it is no longer meaningful in two dimensions).

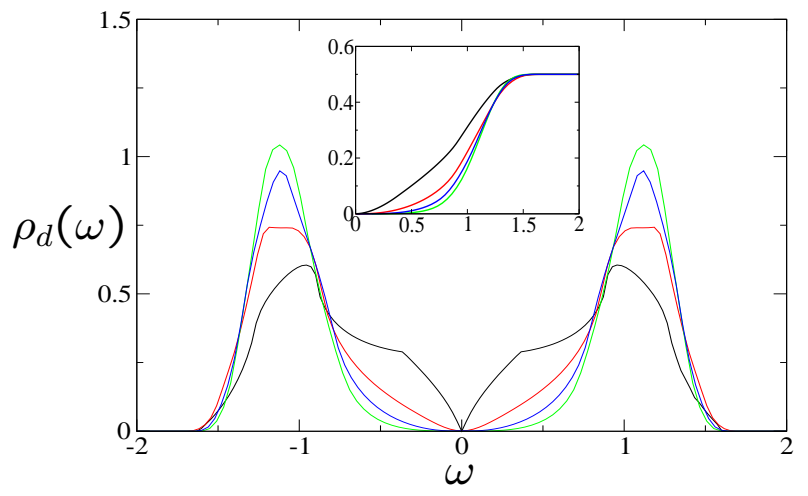

FIG. 12: Local spectral density at the critical point $U=U_{c}$, for increasing dimensionality (top to bottom curves at small $\omega: d=2,3,4,5)$. The spectral weight associated with the low-frequency tails of the Hubbard bands is seen to decrease as dimensionality increases. Correspondingly, the separation of energy scales and the pre-formed gap become more and more apparent. The inset shows the integrated density of states $\int_{0}^{\omega} \mathrm{d} \epsilon \rho_{d}(\epsilon)$ demonstrating this approximate separation of scales for $d \geq 3$. (Note that the progressive narrowing of the main lobe of the Hubbard band as $d$ increases is an artefact of the approximation in which spinons and rotors are decoupled.

\section{CONCLUSION AND PERSPECTIVES}

In this paper, we have used the slave-rotor representation in order to construct approximation schemes for strongly correlated fermion models. A theory of the Hubbard model involving free fermionic spinons selfconsistently coupled to a quantum XY model has been developed. The quantum phase transition of the latter corresponds to the Mott transition between a strongly correlated metal and a Mott insulating spin-liquid with a spinon Fermi surface. Both the gap in the spectral function and the quasiparticle weight vanish at the critical point, while the effective mass remains finite. In this picture, long-wavelength collective modes of the phase variable play an important role. They are responsible for low-energy tails of the Hubbard bands (in addition to the main component of these bands, which are atomiclike short-distance excitations). This has potential implications for spectroscopic and tunneling experiments. In infinite dimensions, these collective modes are suppressed and this limit appears singular in this respect. Only in this limit is a strict separation of energy scales recovered, as in the DMFT picture.

This raises some open questions associated with the physics of these collective modes, which are physically associated with zero-sound. At the saddle-point level, where spinons and rotors do not interact, these modes are undamped. The metallic state is described as a perfect metal with no inelastic scattering. Interactions between rotors and fermionic spinons will induce a Landau damping of these modes, and the metallic state will loose phase coherence. One possibility is that this damping is large, which would presumably weaken its effect and might restore some separation of energy scales as in the DMFT picture. This calls for further work on the nature of collective modes in a metal close to the Mott transition. In particular, the restauration of gauge symmetry, broken at the saddle-point level, will have a strong impact on the non-gauge invariant propagators, as already known for single impurity models [34]. However, it is yet not entirely clear whether this effect will affect strongly the physical sector at low energy. One additional issue is that in a real material the screening deteriorates as one gets closer to the Mott insulating state [35]. The "acoustic plasmon" mode of the Hubbard model with short-range interactions will be pushed to higher energy and this may also weaken its relevance for low-energy physics. Another issue which will arise when taking into account the interactions between spinons and rotors is the description of the insulator as a non-magnetic spin-liquid. Stabilizing such a spin-liquid state beyond saddle-point level is presumably possible only on a very frustrated lattice. Even in this case however, it has been suggested recently 36 that a superconducting phase can intercalate between the metal and the insulator, due to the proliferation of short range spin singlets, therefore superseding the zero temperature Mott transition. Nevertheless, some of our results, such as the finiteness of the effective mass, should remain valid above the low temperature ordered regions.

Finally, we point out that the slave rotor representation explored here is a useful technical tool that can be applied to strongly correlated systems in a variety of contexts. Because a single collective variable is introduced, (which has a direct physical interpretation in connection with the local charge), using this representation is generally simpler than other finite-U slave-boson schemes provided one deals with a symmetric interaction. Ap- 
plications to mesoscopic devices and quantum impurity models have been presented elsewhere 7, 8]. Other potential applications are the effect of long-range or timedependent interactions [22], or the interplay of disorder and interactions. Interacting boson models can also be expressed with slave rotors (for a recent application of variational approximations to the XY model, in the context of bosonic models see 37]). Although mean-field approximations for interacting bosons can be formulated in a simple manner due to the commuting nature of the physical degrees of freedom [29], the slave-rotor representation might prove useful in the context of interacting cold atoms in order to deal, for example, with bosonfermion mixtures.

\section{APPENDIX A: LARGE-N LIMITS AND MEAN-FIELD APPROXIMATIONS}

We discuss here how the different mean field approximations presented in this paper can be formulated in terms of large $N$ limits of generalized Hubbard models. Let us introduce a "multichannel" version of the Hubbard model, based on spin-carrying fermions $f_{\sigma}^{\dagger}(\sigma=$ $1, \cdots, N)$ and channel-carrying phases $\theta_{\alpha}(\alpha=1 \ldots K)$ :

$H=\sum_{\mathrm{i} \sigma} \epsilon_{0} f_{\mathrm{i} \sigma}^{\dagger} f_{\mathrm{i} \sigma}+\frac{U}{2} \sum_{\mathrm{i} \alpha} \hat{L}_{\mathrm{i} \alpha}^{2}-\frac{1}{K} \sum_{\mathrm{ij} \sigma \alpha} t_{\mathrm{ij}} f_{\mathrm{i} \sigma}^{\dagger} f_{\mathrm{j} \sigma} e^{i\left(\theta_{\mathrm{i} \alpha}-\theta_{\mathrm{j} \alpha}\right)}$

Two Hubbard-Stratonovich fields conjugate to $\sum_{\sigma} f_{\mathrm{i} \sigma}^{\dagger} f_{\mathrm{j} \sigma}$ and $\sum_{\alpha} e^{i\left(\theta_{\mathrm{i} \alpha}-\theta_{\mathrm{j} \alpha}\right)}$ can be introduced in order to decouple the last term. When both $N$ and $K$ are large, with a fixed ratio $K / N$, a saddle-point applies which leads to the decoupled effective Hamiltonians (33.34). This corresponds to a factorization the hopping term on bonds, as shown by the effective parameters (35). A similar re- mark applies in the usual context of slave-bosons for the $t-J$ model: the mean-field approximation investigated e.g in 24] correspond to a multichannel limit of:

$$
-\frac{1}{K} \sum_{i j \sigma \alpha} t_{i j} f_{\mathrm{i} \sigma}^{\dagger} f_{\mathrm{j} \sigma} b_{i \alpha} b_{j \alpha}
$$

Because the quantum XY model on the lattice is not easily investigated analytically, we have performed in section IVB a $\mathrm{O}(2 \mathrm{M})$ generalization of the phase part that leads to further simplications, while allowing to deal with the model in finite dimensions. This can also be seen as a direct large $N, \mathrm{M}$ limit of a $\mathrm{SU}(N) \times \mathrm{O}(2 \mathrm{M})$ Hubbard model (see 7] for a related approximation concerning the Anderson model):

$H=\sum_{\mathrm{i} \sigma} \epsilon_{0} f_{\mathrm{i} \sigma}^{\dagger} f_{\mathrm{i} \sigma}+\frac{U}{2 M} \sum_{\mathrm{i} \alpha \beta}\left(\hat{L}_{\mathrm{i}}^{\alpha \beta}\right)^{2}-\sum_{\mathrm{i} j \sigma \alpha} \frac{t_{\mathrm{ij}}}{M} f_{\mathrm{i} \sigma}^{\dagger} f_{\mathrm{j} \sigma} X_{\mathrm{i} \alpha}^{*} X_{\mathrm{j} \alpha}$

introducing a complex field with $\mathrm{M}$ colors $X_{\mathrm{i} \alpha}$ subjected to the spherical constraint: $\sum_{\alpha}\left|X_{\mathrm{i} \alpha}\right|^{2}=M$. In the previous expression, $\hat{L}_{\mathrm{i}}^{\alpha \beta}$ denotes the $\mathrm{O}(2 \mathrm{M})$ angular momentum tensor associated with the $X_{\mathrm{i} \alpha}$ vector 29$]$.

Finally, we note that the simplest single-site mean field of section generalized Hubbard model which reads (note the different indices position and the scaling of the hopping term):

$H=\sum_{\mathrm{i} \sigma} \epsilon_{0} f_{\mathrm{i} \sigma}^{\dagger} f_{\mathrm{i} \sigma}+\frac{U}{2} \sum_{\mathrm{i} \alpha} \hat{L}_{\mathrm{i} \alpha}^{2}-\frac{1}{K^{2}} \sum_{\mathrm{i} j \sigma \alpha \alpha^{\prime}} t_{\mathrm{ij}} f_{\mathrm{i} \sigma}^{\dagger} f_{\mathrm{j} \sigma} e^{i\left(\theta_{\mathrm{i} \alpha}-\theta_{\mathrm{j} \alpha^{\prime}}\right)}$

This gives a on site factorization of the phase variables. Alternatively, this can be seen as a large connectivity of the bond mean field approximation (33 34).
[1] M. Imada, A. Fujimori, and Y. Tokura, Rev. Mod. Phys. 70, 1039 (1998), and references therein.

[2] A. Georges, G. Kotliar, W. Krauth, and M. Rozenberg, Rev. Mod. Phys. 68, 13 (1996), and references therein.

[3] K. Held, I. A. Nekrasov, N. Blümer, V. I. Anisimov, and D. Vollhardt, Int. J. Mod. Phys. B 15, 2611 (2001), condmat/0010395, and references therein.

[4] A. I. Lichtenstein, M. I. Katsnelson, and G. Kotliar, in Electron Correlations and Materials Properties 2, edited by A. Gonis (Kluwer, New York, 2002), condmat/0211076, and references therein.

[5] A. Georges, in Lectures on the physics of highly correlated electron systems, edited by A. Avella and F. Mancini (American Institute of Physics, 2004), condmat/0403123, and references therein.

[6] T. Maier, M. Jarrell, T. Pruschke, and M. H. Hettler (2004), cond-mat/0404055 and references therein.

[7] S. Florens and A. Georges, Phys. Rev. B 66, 165111 (2002).
[8] S. Florens, P. S. Jose, F. Guinea, and A. Georges, Phys. Rev. B 68 (2003).

[9] G. Kotliar and A. E. Ruckenstein, Phys. Rev. Lett. 57, 1362 (1986).

[10] H. Hasegawa, Phys. Rev. B 56, 1196 (1997).

[11] S. Florens, A. Georges, G. Kotliar, and O. Parcollet, Phys. Rev. B 66, 205102 (2002).

[12] L. Perfetti, A. Georges, S. Florens, S. Biermann, S. Mitrovic, Y. Tomm, H. Höchst, and M. Grioni, Phys. Rev. Lett. 90, 166401 (2003).

[13] S. Florens, Ph.D. thesis, Université Paris 6 and Ecole Normale Supérieure, Paris (2003), http://www.lpt.ens.fr/ florens.

[14] R. Fresard and G. Kotliar, Phys. Rev. B 56, 12909 (1997).

[15] J. Bünemann, W. Weber, and F. Gebhard, Phys. Rev. B 57, 6896 (1998).

[16] H. grabert, Phys. Rev. B 50, 17364 (1994).

[17] H. Schoeller and G. Schön, Phys. Rev. B 50, 18436 
(1994).

[18] E. Lebanon, A. Schiller, and F. B. Anders, Phys. Rev. B 68, 041311 (2003).

[19] C. P. Herrero, G. Schn, and A. D. Zaikin, Phys. Rev. B 59, 5728 (1999).

[20] P. Coleman, Phys. Rev. B 29, 3035 (1984).

[21] G. Kotliar, The large $N$ expansion in the strong correlation problem (Elsevier, 1991), les Houches Summer School.

[22] S. Florens, L. de' Medici, and A. Georges (2004), unpublished.

[23] A. Hewson, The Kondo problem to heavy fermions (Cambridge, 1996).

[24] G. Kotliar and J. Liu, Phys. Rev. B 38, 5142 (1988).

[25] I. Affleck and B. Marston, Phys. Rev. B 39, 11538 (1989).

[26] J. P. Lu, Phys. Rev. B 49, 5687 (1994).

[27] W. F. Brinkman and T. M. Rice, Phys. Rev. B 2, 4302 (1970).

[28] M. P. A. Fisher, P. B. Weichman, G. Grinstein, and D. S. Fisher, Phys. Rev. B 546, 40 (1989).

[29] S. Sachdev, Quantum Phase Transitions (Cambridge, 2000).

[30] E. H. Lieb and F. Y. Wu, Phys. Rev. Lett. 20, 1445 (1968).

[31] D. P. Arovas and A. Auerbach, Phys. Rev. B 38, 316
(1988).

[32] J. Hubbard, Proc. Roy. Soc. A 281, 401 (1964).

[33] C. Castellani, G. Kotliar, R. Raimondi, M. Grilli, Z. Wang, and M. Rozenberg, Phys. Rev. Lett. 69, 2009 (1992).

[34] P. Coleman, Phys. Rev. B 35, 5072 (1987).

[35] N. F. Mott, Phil. Mag. 6, 287 (1961).

[36] M. Capone, M. Fabrizio, C. Castellani, and E. Tosatti (2004), cond-mat/0401090.

[37] J. J. Garcia-Ripoll, J. I. Cirac, P. Zoller, C. Kollath, U. Schollwoeck, and J. von Delft (2003), condmat/0306162.

[38] R. Assaraf, P. Azaria, M. Caffarel, and P. Lecheminant, Phys. Rev. B 60, 2299 (1999).

[39] A rescaling of the Coulomb repulsion $U \rightarrow U / 2$ was used in order to preserve the exact atomic limit, as discussed in [7]

[40] In the spherical X-field approximation, a finite $U_{c}$ is not possible in $d=1$ because of the Mermin-Wagner theorem. Had we kept the $\mathrm{O}(2)$ rotor variable, we would be able to correctly describe the Berezinskii-KosterlitzThouless nature of the Mott transition, even in the approximation of decoupled spinons and rotors. A finite $U_{c}$ is possible in $d=1$, as indeed found for larger orbital degeneracy, see e.g the analysis of the case $N=4$ in [38]. 